\title{
Optimal Placement of Unified Power Flow Controller for Minimization of Power Transmission Line Losses
}

\author{
Sreerama Kumar R., Ibrahim M. Jomoah, and Abdullah Omar Bafail
}

\begin{abstract}
This paper proposes the application of genetic algorithm for the determination of the optimal placement of unified power flow controller (UPFC) in a power system so as to minimize the system losses and enhance the voltage profile. The method can identify the line in which the UPFC can be located. UPFC is represented as a decoupled synchronous voltage source model in the conventional Newton-Raphson load flow NRLF algorithm. The method of interface of the UPFC model with the NRLF algorithm is based on the fact that the insertion of UPFC in a transmission line is equivalent to adding a new $P Q$ bus in the system, and hence the real and reactive power mismatches corresponding to this bus are computed after appropriately modifying the line and bus data. The genetic algorithm proceeds with an initial randomly generated population. The effectiveness of each of the individuals in the population is evaluated on the basis of a fitness function defined to reflect the effect of the real power losses in the system. Preliminary investigations indicate the effectiveness of this approach in minimizing the transmission line losses and the enhancement of the voltage profile.
\end{abstract}

Index Terms - Genetic algorithm, loss minimization, power system steady state simulation, UPFC model interface.

\section{INTRODUCTION}

As the demand for electrical energy is continuously increasing, it necessitates the expansion of the transmission network along with the installation of new power plants. But at the same time, such projects are getting delayed or even blocked due to many factors such as cost and environmental considerations and also the difficulties in getting the right of way over long distances. Hence effective utilization of the existing network to their maximum possible operational limits is important. Minimization of system losses and thus the enhancement of power transfer capability of the transmission network has always been a concern in power supply industry. Congestion in Power flow in strategic routes may occur because of the inflexibility of their power control capability. From this perspective, appropriate application of the flexible AC transmission systems (FACTS) technologies offer methods for better power flow control and better utilization of the transmission networks in power systems [1]-[3]. Unified power flow controller (UPFC) is the one of the most advanced FACTS devices capable of providing better power transfer capability and better voltage profile in the system [3]. Once the rating of UPFC for the enhancement of transmission network is determined, it is necessary to identify optimal locations of the UPFCs in the transmission

Manuscript received November 11, 2013; revised January 14, 2014.

The authors are with the Vice Presidency for Projects, King Abdulaziz University, Jeddah, P.O. Box: 80200, Jeddah 21589, Saudi Arabia (e-mail: \{skramdas, ijomaah, abafail\} @kau.edu.sa). system so as to meet the specified objectives without violating the operational constraints. There are many optimization techniques which can be applied for this problem. Genetic algorithm [4]-[6] is an optimization tool which starts with an assumed solution space, and proceeds to generate better and better off-springs based on the principle of survival of the fittest.

This paper proposes a genetic algorithm based method for finding the optimal location of UPFCs to be installed in a power system, for the minimization of the system losses and at the same time meeting the operational constraints on line flows and bus voltages. UPFC is represented as a synchronous voltage source model so as to interface with the conventional Newton-Raphson load flow (NRLF) algorithm. Preliminary investigations indicate that this method is effective for determining the optimal location of the UPFCs for improving the steady state performance of the power systems.

The rest of the paper is organized as follows: The details of UPFC model and the method of interface with NRLF are given in Section II. The application of genetic algorithm for finding the optimal UPFC location so as to minimize the system losses is discussed in Section III. Test system details and simulation results are given in the following section and finally the conclusion.

\section{UPFC MODEL AND INTERFACE WITH NRLF ALGORITHM}

\section{A. UPFC Model}

As shown in Fig. 1, the basic structure of UPFC consists of a set of AC to DC voltage source converters connected back to back and operated from a common DC link capacitor [1]. As given in Fig. 1, one of the converters is connected in shunt and the other in series with the line. The shunt converter, $\mathrm{VSC}_{1}$, is used to provide real power demand of the series converter through a common DC link. It can also generate or absorb reactive power, if it is desired, and thereby provide independent shunt reactive compensation for the line. The series converter, $\mathrm{VSC}_{2}$, of the UPFC injects a voltage with controllable magnitude and phase angle in series with the line. Fig. 2 shows a UPFC inserted in line $m-n$. The scheduled line flow measured at Bus $m$ is $\left(P_{m n}+j Q_{m n}\right)$. The $\left(P_{m} j Q_{m}\right)$ and $\left(P_{n}, j Q_{n}\right)$ are the load power specified at buses $\mathrm{m}$ and $\mathrm{n}$ respectively. The UPFC bus is represented as a PQ bus as shown in Fig. 3. In this model, the fictitious bus, Bus $\mathrm{U}$, is introduced to maintain the scheduled power flow $\left(P_{m n}+\right.$ $j Q_{m n}$ ) in line $m-n$. In this model, losses in the UPFC are ignored. A simplification of the UPFC model given in Fig. 3 is obtained by neglecting the impedance of the coupling transformers. In this situation, the shunt converter can be 
assumed to operate at unity power factor and the UPFC is represented by an ideal series voltage source. This model is referred to as synchronous voltage source UPFC model [3]. If the losses in UPFC converter and transformers are assumed to be negligible, UPFC can be modeled by transforming Bus $\mathrm{m}$ into a PV bus and Bus U into a PQ bus as shown in Fig. 4.
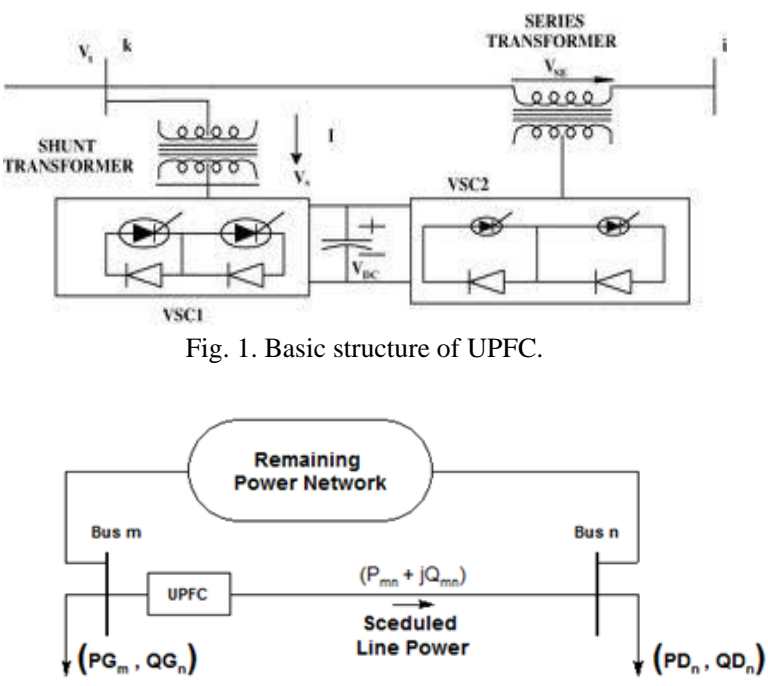

Fig. 2. UPFC inserted in line $m-n$.

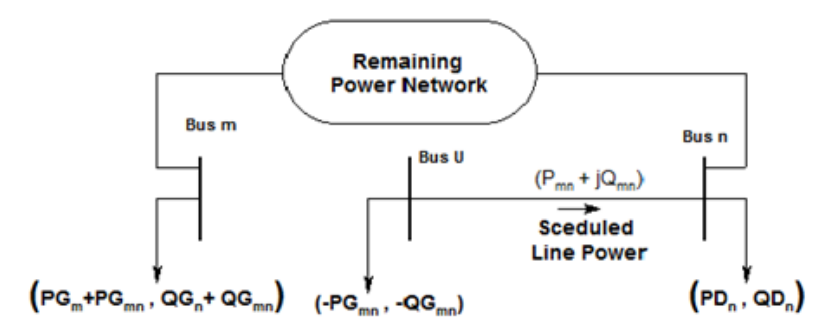

Fig. 3. UPFC Bus modeled as a PQ bus.

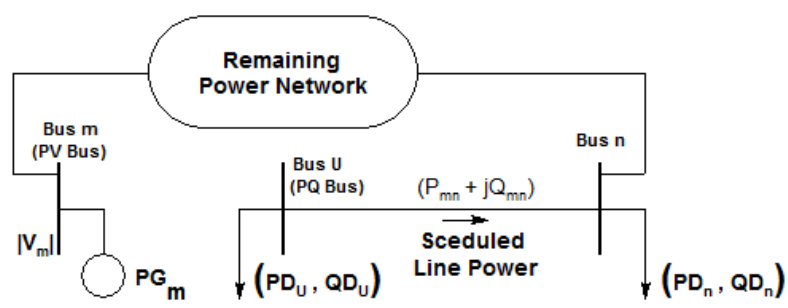

Fig. 4. Decoupled synchronous voltage source Model of UPFC.

\section{B. Newton-Raphson Load Flow Algorithm}

Load flow analysis involves the determination of the bus voltages, power injections at all the buses and power flows though the transmission network for a specified load demand at various buses in the system. As the load flow problem involves the simultaneous solution of non-linear algebraic equations, the solution technique is iterative in nature. NRLF algorithm is suited to large practical power systems specifically because of its quadratic convergence properties. Further, the number of iterations required for a specified accuracy is significantly less than the other techniques and are practically independent of the system size. The conventional NRLF problem formulation is given by [7]

$$
\left[\begin{array}{cc}
H & N \\
J & L
\end{array}\right]\left[\begin{array}{c}
\Delta \delta \\
\Delta V / V
\end{array}\right]=\left[\begin{array}{c}
\Delta P \\
\Delta Q
\end{array}\right]
$$

The $H, N, J$ and $L$ are the sub-matrices of the Jacobian matrix. Expressions for the Jacobian elements can be obtained by partial differentiation of equations (2) and (3). The power flow solutions are usually started with a flat voltage start assumption of 1 p.u. for the bus voltage magnitudes and zero degrees for bus voltage angles. With these values, the real and reactive powers at Bus $K$ are calculated for all buses as

$$
\begin{gathered}
P_{K \text { calculated }}=\sum_{M=1}^{N B} V_{K} V_{M} \quad\left(G_{K M} \cos \delta_{K M}+B_{K M} \sin \delta_{K M}\right) \\
\text { for } K=1,2, \ldots, N B \\
Q_{K \text { calculated }}=\sum_{M=1}^{N B} V_{K} V_{M} \quad\left(G_{K M} \sin \delta_{K M}-B_{K M} \cos \delta_{K M}\right) \\
\text { for } K=1,2, \ldots, N B
\end{gathered}
$$

Then the bus power mismatches are

$$
\begin{aligned}
& \Delta P_{K}=P_{K}(\text { specified })-P_{K}(\text { calculated }) \\
& \Delta Q_{K}=Q_{K}(\text { specified })-Q_{K}(\text { calculated })
\end{aligned}
$$

All the elements of the Jacobian matrix are computed and the NRLF equations are solved for the corrections in bus voltage magnitudes $(\Delta V / V)$ and bus voltage angles $(\Delta \delta)$. Modified values for bus voltage magnitudes and voltage angles are obtained by adding the corrections to the respective assumed values. The iterations are repeated till $\left|\Delta P_{K}\right| \leq \varepsilon$ and $\left|\Delta Q_{K}\right| \leq \varepsilon$ for all $i$ where $\varepsilon$ is the desired tolerance.

\section{UPFC Interfacing Approach}

Each UPFC causes the addition of a PQ bus, with corresponding $\Delta \mathrm{P}$ and $\Delta \mathrm{Q}$ mismatch equations of the NRLF algorithm. The buses between which UPFC is connected is determined. A PQ bus is introduced between those two buses. Then the line data and bus data are modified accordingly. For each additional row and column of the Jacobian matrix due to a UPFC, there is a corresponding additional power mismatch equation that must be satisfied in order for the NRLF to converge to the solution. Since these equations are similar in form to the original power mismatch equations the overall convergence characteristics are largely unaffected. The state variables corresponding to the UPFC are combined with the network bus voltage magnitudes and angles in a single frame-of-reference for a unified solution through the NRLF algorithm. The UPFC state variables are adjusted automatically so as to satisfy specified power flows and voltage magnitudes.

\section{ApPliCAtion OF GENETIC AlgORITHM FOR OPTIMAL UPFC PLACEMENT}

GAs are global search techniques based on the mechanism of natural selection and genetics [5]. Without any prior knowledge of the objective function, GAs can search several possible solutions simultaneously. GA starts with random generation of initial population and then the selection, crossover and mutation operations are carried out until best 
population is arrived at. In the application of GA for the determination of the UPFC location in a power system for the minimization of transmission line losses, the buses between which UPFC can be connected are considered as the variables. Considering the bounds and the resolution of these variables, the first population of these variables is generated at random. This population is worked upon by the genetic operators such as reproduction, crossover and mutation. Load flow analysis with the incorporation of the UPFC model is used for the calculation of fitness function of each individual in the first population. The fitness function is chosen as

$$
F n=1.0 /\left(1+P_{\text {Loss }}\right)
$$

where $P_{\text {Loss }}$ is the total transmission line losses in the system given by

$$
\begin{gathered}
P_{\text {Loss }}=\sum_{M=1}^{N B} G_{K M}\left\{V_{K}^{2}+V_{M}^{2}-2 V_{K} V_{M} \operatorname{Cos}\left(\partial_{K M}\right)\right. \\
\text { for } K=1,2, \ldots, N B
\end{gathered}
$$

Each individual is taken up from the population. The necessary modifications in line and bus data because of the insertion of UPFC are made and the load flow analysis is performed. This is followed by the calculation of the fitness function for that particular individual. The individual with maximum fitness value has the highest preference to be selected for the next generation as it will occupy a major portion on the roulette wheel. The selected individuals are subjected to crossover and mutation operations. A single point crossover is applied. A new population is obtained after crossover and mutation. The fitness value of each individual is calculated in the same manner as for the initial population. Again these individuals are acted upon by the operators. According to the maximum number of generations specified, the process is repeated to obtain the best fit individual. GA provides a number of potential solutions to a problem and the choice of final solution is left to the user. In cases where a particular problem does not have one individual solution, then the GA is potentially useful for identifying these alternative solutions simultaneously.

\section{Test System AND Simulation Results}

The effectiveness of the proposed approach for the optimal location of UPFC is investigated on the test system shown in Fig. 1. The system data required for load flow analysis of systems with UPFC is given in Table I and Table II respectively. The UPFC is used to maintain active and reactive powers leaving the UPFC at $40 \mathrm{MW}$ and $2 \mathrm{MVAR}$ respectively. In addition to the load flow and the UPFC data, the inputs that are to be provided include population size, number of generations, cross over probability, mutation probability, number of variables, resolution of variables and variable bounds. The buses between which UPFC can be connected are considered as the variables in this problem. Considering the bounds and the resolution of these variables, the first population of these variables is generated. For the five bus test system shown in Fig. 1, these variables range from bus numbers 2 to 5 as it is assumed not to place UPFC near slack bus which is numbered as 1 . So, the upper bound of the variables is 2 and lower bound 5. The resolution of the variables in this case is 1 . The population size is taken as 30 , A random initial population consisting of 30 individuals is created. The crossover probability is assumed to be 0.98 and the mutation probability.001.

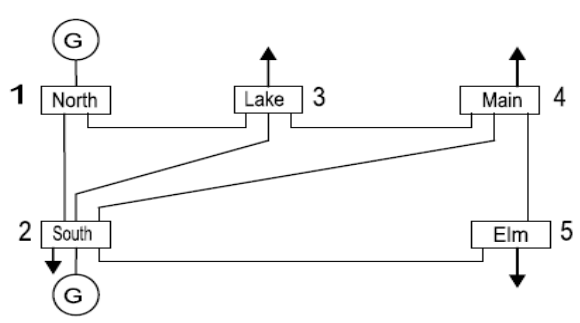

Fig. 5. Five bus standard test system.

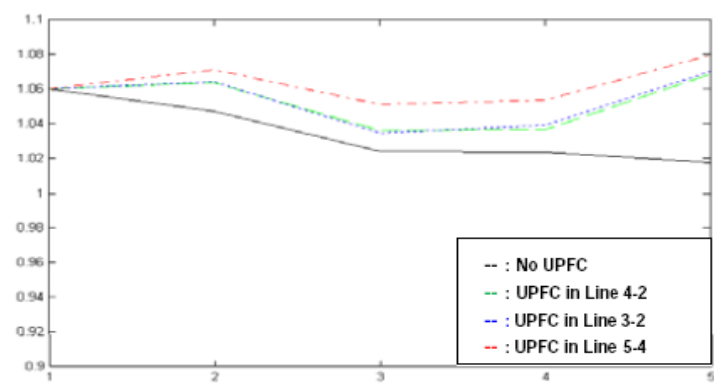

Fig. 6. Bus voltage profile of the five bus system with UPFC at different locations.

\begin{tabular}{|c|c|c|c|c|}
\hline $\begin{array}{c}\text { Start } \\
\text { Bus }\end{array}$ & $\begin{array}{c}\text { End } \\
\text { Bus }\end{array}$ & $\begin{array}{c}\text { Resistance } \\
\text { (P.U.) }\end{array}$ & $\begin{array}{c}\text { Reactance } \\
\text { (P.U.) }\end{array}$ & $\begin{array}{c}\text { Half-Line } \\
\text { Charging } \\
\text { (P.U.) }\end{array}$ \\
\hline 1 & 2 & 0.02 & 0.06 & 0.030 \\
\hline 1 & 3 & 0.08 & 0.24 & 0.025 \\
\hline 2 & 3 & 0.06 & 0.18 & 0.020 \\
\hline 2 & 4 & 0.06 & 0.18 & 0.020 \\
\hline 2 & 5 & 0.04 & 0.12 & 0.015 \\
\hline 3 & 4 & 0.01 & 0.03 & 0.010 \\
\hline 4 & 5 & 0.08 & 0.24 & 0.025 \\
\hline
\end{tabular}

The results of the load flow analysis of the test system without the incorporation of UPFC are given in Table III. The original network is then modified to include a UPFC, which compensates the transmission line associated with it. As per the model used, the introduction of UPFC is equivalent to adding a new PQ node. The UPFC is used to maintain active and reactive powers leaving the UPFC, towards the farthest bus. Moreover, the UPFC's shunt converter is set to regulate nearest voltage magnitude of its nearest bus. The application of GA to the problem indicate that the best UPFC location satisfying the condition of minimum line losses occurs when it is placed in the transmission line between buses 5 and 4 , nearer to bus 5. Table IV shows the results of the load flow analysis of the test system with the incorporation of UPFC. Voltage profile of the system with UPFC included in various lines is shown in Fig. 5. It can be seen that the voltage profile is the best when the UPFC is in line 5-4, placed nearer to bus 5. Table $\mathrm{V}$ shows a comparison of the line losses with the UPFC in the optimal location against the case without UPFC. The Table indicates that the UPFC it its optimal location could reduce the line losses in the system by $70 \%$ when compared to the system without UPFC (see Fig. 6). 
TABLE II: BUS DATA

\begin{tabular}{|c|c|c|c|c|c|c|}
\hline \multirow{2}{*}{ Bus No. } & \multicolumn{2}{|c|}{ Bus Voltage } & \multicolumn{2}{c|}{ Power Generation } & \multicolumn{2}{c|}{ Power Demand } \\
\cline { 2 - 7 } & $\begin{array}{c}|V| \\
(\text { P.U.) }\end{array}$ & $\begin{array}{c}\delta \\
(\mathrm{Deg})\end{array}$ & $\begin{array}{c}P_{G} \\
(\mathrm{MW})\end{array}$ & $\begin{array}{c}Q_{G} \\
\text { (MVAR) }\end{array}$ & $\begin{array}{c}P_{D} \\
(\mathrm{MW})\end{array}$ & $\begin{array}{c}Q_{D} \\
\text { (MVAR) }\end{array}$ \\
\hline 1 & 1.06 & 0.0 & -- & -- & -- & -- \\
\hline 2 & 1.00 & 0.0 & 40 & 30 & 20 & 10 \\
\hline 3 & -- & -- & -- & -- & 45 & 15 \\
\hline 4 & -- & -- & -- & -- & 40 & 5 \\
\hline 5 & -- & -- & -- & -- & 60 & 10 \\
\hline
\end{tabular}

TABLE III: RESULTS OF LOAD FLOW ANALYSIS WITHOUT UPFC

MAXIMUM POWER MISMATCH $=1.70419 \mathrm{E}-014$, NO. OF ITERATIONS $=5$

\begin{tabular}{|c|c|c|c|c|c|c|}
\hline \multirow{2}{*}{$\begin{array}{l}\text { Bus } \\
\text { No. }\end{array}$} & \multicolumn{2}{|c|}{ Bus Voltage } & \multicolumn{2}{|c|}{ Power Generation } & \multicolumn{2}{|c|}{ Power Demand } \\
\hline & $\begin{array}{c}|V| \\
\text { (P.U.) }\end{array}$ & $\begin{array}{c}\delta \\
\text { (Deg) }\end{array}$ & $\begin{array}{c}P_{G} \\
(\mathrm{MW})\end{array}$ & $\begin{array}{c}Q_{G} \\
\text { (MVAR) }\end{array}$ & $\begin{array}{c}P_{D} \\
(\mathrm{MW})\end{array}$ & $\begin{array}{c}Q_{D} \\
\text { (MVAR) }\end{array}$ \\
\hline 1 & 1.060 & 0.00 & 129.59 & -7.42 & 0.00 & 0.00 \\
\hline 2 & 1.047 & -2.8 & 40.00 & 30.00 & 20.00 & 10.0 \\
\hline 3 & 1.024 & -4.50 & 0.00 & 0.00 & 45.00 & 15.0 \\
\hline 4 & 1.024 & -5.33 & 0.00 & 0.00 & 40.00 & 5.00 \\
\hline 5 & 1.018 & -6.15 & 0.00 & 0.00 & 60.00 & 10.0 \\
\hline & \multicolumn{2}{|c|}{ Total } & 169.58683 & 22.57891 & 165.00000 & 40.00000 \\
\hline
\end{tabular}

TABLE IV: RESUlTS OF LOAD FLOW ANALYSIS WITH UPFC IN LINE 5-4 NEAR BUS 5 MAXIMUM POWER MISMATCH $=8.52096 \mathrm{E}-015$, NO. OF ITERATIONS $=5$

\begin{tabular}{|c|c|c|c|c|c|c|}
\hline \multirow{2}{*}{$\begin{array}{l}\text { Bus } \\
\text { No. }\end{array}$} & \multicolumn{2}{|c|}{ Bus Voltage } & \multicolumn{2}{|c|}{ Power Generation } & \multicolumn{2}{|c|}{ Power Demand } \\
\hline & $\begin{array}{c}|V| \\
\text { (P.U.) }\end{array}$ & $\begin{array}{c}\delta \\
\text { (Deg) }\end{array}$ & $\begin{array}{c}P_{G} \\
(\mathrm{MW})\end{array}$ & $\begin{array}{c}Q_{G} \\
\text { (MVAR) }\end{array}$ & $\begin{array}{c}P_{D} \\
(\mathrm{MW})\end{array}$ & $\begin{array}{c}Q_{D} \\
\text { (MVAR) }\end{array}$ \\
\hline 1 & 1.06 & 0.00 & 26.37 & -30.66 & 0.00 & 0.00 \\
\hline 2 & 1.07 & -0.47 & 40.00 & 30.00 & 20.00 & 10.00 \\
\hline 3 & 1.05 & -2.35 & 0.00 & 0.00 & 45.00 & 15.00 \\
\hline 4 & 1.05 & -2.31 & 0.00 & 0.00 & 40.00 & 5.00 \\
\hline 5 & 1.08 & 0.43 & 0.00 & 0.00 & -40.00 & -2.00 \\
\hline & \multicolumn{2}{|c|}{ Total } & 66.37 & -0.66 & 65.00 & 28.00 \\
\hline
\end{tabular}

TABLE V: EFFECT OF UPFC ON LINE LOSSES

\begin{tabular}{|c|c|c|c|c|c|}
\hline \multicolumn{2}{|c|}{ Bus No. } & \multicolumn{3}{c|}{ Line Loss } & \multicolumn{2}{c|}{ Line Loss } \\
\multicolumn{2}{|c|}{} & \multicolumn{2}{c|}{ With UPFC in Line 5-4 near } \\
Bus 5 \\
\hline Start Bus & End Bus & MW & MVAR & MW & MVAR \\
\hline 1 & 2 & 1.41 & -2.43 & 0.10 & -6.51 \\
\hline 1 & 3 & 1.19 & -1.86 & 0.24 & -4.84 \\
\hline 2 & 3 & 0.35 & -3.24 & 0.27 & -3.69 \\
\hline 2 & 4 & 0.44 & -2.97 & 0.25 & -3.77 \\
\hline 2 & 5 & 1.13 & 0.18 & 0.09 & -3.20 \\
\hline 3 & 4 & 0.04 & -1.99 & 0.01 & -2.19 \\
\hline 4 & 5 & 0.03 & -5.12 & 0.41 & -4.46 \\
\hline
\end{tabular}

\section{Conclusions}

This paper has proposed a methodology based on genetic algorithm for the minimization of system transmission line losses by the optimal placement of UPFC. UPFC has been modeled as a decoupled synchronous voltage source model which is interfaced with the conventional NRLF algorithm. The load flow analysis has been performed for each individual in every generation so as to determine the fitness function which is defined as the inverse of the total transmission line losses. The location of UPFC has been identified as the best individual from among the off-springs of the last generation. The simulation results on a standard test system indicate that the installation of UPFC at optimal locations in the system can lead to significant reduction of transmission losses and also the improvement of the bus voltage profile of the system. Further, genetic algorithm approach has seen to be a potential technique with 
mathematically less complex than that of the conventional optimization techniques. The extension of this research to practical large power systems is under progress.

\section{REFERENCES}

[1] N. G. Hingorani and L. Gyugyi, Understanding FACTS - Concepts and Technology of Flexible AC Transmission Systems, IEEE Press, New York, 1999.

[2] C. R. F. Esquivel and E. Acha, "A critical comparison of newton-raphson UPFC algorithms in power flow studies," in Proc. IEE Generation, Transmission and Distribution, vol. 144, no. 5, pp. 437-444, 1997.

[3] D. J. Gotham and G. T. Heydt, "Power flow control and power flow studies for systems with FACTS devices," IEEE Transactions on Power Systems, vol. 13. no.1, pp. 60-65, 1997.

[4] V. Krishnasamy, "Genetic algorithm for solving optimal power flow problem with UPFC," International Journal of Software Engineering and Its Applications Ts Applications, vol. 5, no. 1, pp. 39-50, January, 2011.

[5] D. E. Goldberg, Genetic Algorithms in Search, Optimization and Machine Learning, Longman Publishing Co., Inc. Boston, MA, USA: Addison-Wesley, 1989.

[6] L. Hassan, H. Okman, M. Moghavvemi, H. A. F. Almurib, and K. M. Muttaqi, "A coordinated design of PSS and UPFC-Based stabilizer using genetic algorithm," in Proc. IEEE Industry Applications Society Annual Meeting, 2013, pp. 1-9.

[7] H. Saadat, Power System Analysis, 3rd ed. PSA Publishing, 2010.

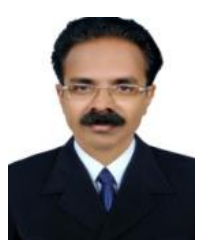

Sreerama Kumar R. is a professor of electrical engineering in King Abdulaziz University, Jeddah, Saudi Arabia. He did his B.Tech in electrical engineering from NSS College of Engineering Palakkad, Kerala, India, M.Tech from Indian Institute of Technology Madras and $\mathrm{Ph} . \mathrm{D}$. from Indian Institute of Science Bangalore, India in 1992 . He is the recipient of the prestigious national award, constituted by the Indian Society for Technical Education, for Promising Engineering Teacher for the year 2003 for creative work done in technical education and the ISTE national award for the best engineering college Teacher of Kerala state in 2008. He is a fellow of the Institution of Engineers (India) and senior member of IEEE. His major fields of interest include modeling and simulation of power system dynamics and control.

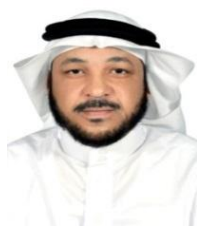

Ibrahim M. Jomoah is the director of Emergency and Disaster Center and associate professor in the Department of Industrial Engineering, King Abdulaziz University (KAU), Saudi Arabia. He did his B.S. from KAU, and then both M.S. and Ph.D. in industrial engineering from the University of Miami in 1990 and 1994 respectively. He is an active researcher in the field of Industrial Engineering for more than 25 years in both governmental and industrial sectors. He is a member of several professional societies and organizations. He has delivered keynote address in several international conferences. He has more than 30 publications to his credit in the fields of Industrial Engineering, Human Factors Engineering, DSM and $\mathrm{EE}$, quality and corporate planning and restructuring.

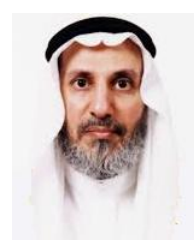

Abdullah Omar Bafail is the vice president for Projects and professor in the Department of Industrial Engineering, King Abdulaziz University, Jeddah, Saudi Arabia. He did his B.Sc. in Systems Engineering from King Fahd University of Petroleum and Minerals, Dhahran, Saudi Arabia, M.Sc. in Industrial and Systems Engineering from University of South California, Los Angeles, USA and Ph.D in the same area from Arizona state University, USA. He is an active researcher in the field of industrial engineering for more than 35 years in both governmental and industrial sectors. He is a member of several professional societies and organizations. His major fields of expertise include dynamic forecasting, quality control, inventory control, simulation and experimental design. 